\title{
Le Rôle De La Responsabilité Sociale Des Entreprises Dans La Gestion Des Risques Psychosociaux: Cas De L'agence Nationale Des Ports
}

\author{
Amina Tourabi, Enseigante chercheure \\ Laboratoire LGII: Equipe du Management Industriel : \\ ENSA Université IBNZOHR Agadir Maroc
}

doi: 10.19044/esj.2016.v12n8p181 URL:http://dx.doi.org/10.19044/esj.2016.v12n8p181

\begin{abstract}
Understood as a key theme in management literature, social responsibility (CSR) is installed between two frames reflections namely business ethics and sustainable development. Several researchers have invested in the issue of CSR but few who have reconciled with the risk management and prevention modalities. By analyzing the social contribution of CSR, our goal is to highlight the emerging relationship between CSR and management of psychosocial risks (RPS), formulating the following questions: can we reconcile CSR as a tool RPS management? What is the contribution of CSR to PR management issues? What is the effect of CSR on factors psychosocial risks? Based on the theoretical foundations of CSR, we propose to cross the methodology and action of CSR in RPS generators factors while controlling the level of well-being at work. By analyzing the perception of the employees of the CSR approach, we presented the maturity level of CSR through the materiality test, then we evaluated the social aspect of the approach through the score method to finally study the differences between the criticality PHI before and after the CSR deployment in port activity.
\end{abstract}

Keywords: Well-being at work, stress, prevention, CSR maturity risk factor

\section{Résumé}

Entendue comme un thème phare dans la littérature managériale, la responsabilité sociale des entreprises (RSE) s'installe entre deux cadres de réflexions à savoir l'éthique des affaires et le Développement Durable. Plusieurs chercheurs ont investit dans la question mais peu sont qui l'ont rapproché avec le management des risques et les modalités de prévention. En analysant l'apport social de la RSE, notre objectif est de mettre en lumière la relation émergente entre la RSE et la gestion des risques psychologiques 
(RPS), en formulant les questions suivantes : peut-t-on concilier la RSE comme un outil de gestion des RPS? Quel est l'apport de la RSE à la problématique de gestion des RPS? Quelle est l'action de la RSE sur les facteurs des RPS? En se basant sur les fondements théoriques de la RSE, nous proposons de croiser la méthodologie et l'action RSE sur les facteurs générateurs de RPS tout en explicitant le niveau de bien être au travail. En analysant la perception des salariés de la démarche RSE, nous avons présenté le niveau de maturité RSE à travers le test de matérialité, puis nous avons évalué le volet social de la démarche à travers la méthode des scores pour enfin étudier les écarts entre les criticités des RPS avant et après le déploiement RSE dans l'activité portuaire.

Mots - clés: Bien être au travail, stress, prévention, maturité RSE, facteur de risques

\section{Introduction}

Entendue comme un thème phare dans la littérature managériale, la responsabilité sociale des entreprises (noté par la suite RSE) s'installe entre deux cadres de réflexions à savoir l'éthique des affaires et le Développement Durable (Mathieu 2008). Bien qu'elle fait aujourd'hui une réflexion de mode, la notion de RSE s'est enraciné depuis les années 50 en proliférant des changements organisationnels complémentaires en faveur d'une compétitivité plus durable au niveau économique, environnemental et social. En pratique, et sous une approche d'interaction et d'interdépendance, elle combine plusieurs disciplines allant des sciences de gestion, du management industriel, de la gestion des ressources humaines, du management de risques etc. ..(Fraisse \& al., 2005).

En analysant l'apport social de la RSE, notre objectif est de mettre en lumière la relation émergente entre la RSE et la gestion des risques psychologiques (noté par la suite RPS), en formulant les questions suivantes : peut-t-on concilier la RSE comme un outil de gestion des RPS, (Leroux \& al., 2011)? Quel est l'apport de la RSE à la problématique de gestion des RPS? Quelle est l'action de la RSE sur les facteurs des RPS?

Plusieurs chercheurs ont investit dans la question de la RSE en la discutant en tant qu'instrument des pratiques de gestion des ressources humaines (noté par la suite GRH) (Windsor 2006) répondant aux exigences des parties prenantes, mais peu sont qui l'ont rapproché avec le management des risques et les modalités de prévention.

En se basant sur ses fondements théoriques de la RSE (Wood 1991), basé sur l'individu et en discutant ses différentes méthodologies sur son volet social (ISO 26000) relevant à la fois des approches préventives et correctives, notre démarche consiste à encadrer les points communs soutenus 
entre démarche RSE et la gestion des RPS et à démontrer que ses préconisations contribuent à la maîtrise des facteurs sources des RPS et à l'amélioration du bien être au travail en général (Leroux et Van de Portal, 2011). Notre investigation empirique a porté sur l'activité portuaire (activité génératrice de stress et d'autres risques psychosociaux) en cherchant à valider les conclusions théoriques au niveau de l'agence nationale des ports (notée par la suite ANP).

Ainsi, après avoir justifié notre démarche, nous passerons en revue les champs théoriques qui ont définis la RSE et son déploiement, puis nous analyserons les processus de gestion des RPS tout en distinguant le champ d'intervention de la RSE avant de passer à l'exploitation des enquêtes réalisées au niveau de l'ANP sur la perception des salariés de la démarche et sur sa contribution à la réduction de leur RPS.

\section{Les fondements théoriques de la RSE}

Si on compte aujourd'hui plus de 37 définitions de la RSE (Carroll \& Shabana, 2010), il n'existe pas à l'heure actuelle un seul modèle académique dominant. En concret, c'est au nouveau courant académique "Business and society" qui s'intéresse aux relations entre entreprise et son environnement qu'on doit l'émergence de la réflexion actuelle sur la RSE. (Acquier et Gond, 2005). Conçue comme un mariage de raison entre la performance sociale et la performance économique, la RSE a suscité vers les années 1960 et 1970 dans la littérature une multitude des définitions (Wood 1991) expliquée par les évolutions théoriques en la matière, accompagnée par d'autres d'ordre politique et environnemental (Benyyedder, \& al,. 2009). Il s'agit en réalité de la concrétisation de l'intégration multidimensionnelle des aspects économiques, éthiques, sociaux et environnementaux (Sautré 2003, Lockert \& al,. 2006). Du côté institutionnel, les définitions sont beaucoup plus axées sur les parties prenantes tout en insistant sur le bien être (Déjean et Gond, 2004, cité par Benydder 2009 p.4), comme cité par la commission des communautés européennes (CCE, 2001, p3): « la RSE est l'intégration volontaire par les entreprises de préoccupations sociales et environnementales à leurs activités commerciales et leurs relations avec leurs parties prenantes ». Cette définition met en place un engagement important de l'entreprise qui prend un caractère obligatoire voire même légal.

En ce qui suit nous exposons les différentes acceptions de la notion depuis son apparition aux Etats Unies jusqu'à son développement en Europe.

\section{RSE à l'américaine, RSE à l'Européenne: différentes acceptations}

Née et développée après une certaine "contingence culturelle", la RSE a toujours constitué un champ prolifique de plusieurs conceptions dans différents moments et contextes. Des auteurs comme (Acquier et coll., 2005 ; 
Doh et Guay, 2006 ; Capron, Quairel- Lanoizelée, 2007) relèvent en fait une dualité existante entre l'approche américaine et celle européenne (Benyedder 2009) comme résumé dans le tableau suivant:

Tableau 1: Conceptions théoriques de la RSE

\begin{tabular}{|c|c|c|c|}
\hline Dimension & RSE à l'américaine & RSE à l'Européenne & $\begin{array}{l}\text { Fondements } \\
\text { théoriques }\end{array}$ \\
\hline Emergence & 1953 & 1995 & Doh et Guay, 2006 \\
\hline Approche & Correction des effets & Prévention des risques & Crépon 2003 \\
\hline $\begin{array}{l}\text { Nature de } \\
\text { l'action }\end{array}$ & Volontaire & Réglementaire & $\begin{array}{l}\text { Capron et Quairel- } \\
\text { Lanoizelée, } 2007 .\end{array}$ \\
\hline Courant & Orthodoxe & Social & $\begin{array}{c}\text { Ben yedder, \& } a l, . \\
2009 .\end{array}$ \\
\hline Origine & Ethiques et religieux & $\begin{array}{l}\text { Développement } \\
\text { durable }\end{array}$ & $\begin{array}{l}\text { Ivanaj et Mc Intyre, } \\
2006\end{array}$ \\
\hline Logique & Individuelle & Institutionnelle (Etat) & $\begin{array}{c}\text { Bollinger et Hofstede, } \\
1987\end{array}$ \\
\hline Critiques & $\begin{array}{l}\text { Entraves au } \\
\text { libéralisme } \\
\text { économique }\end{array}$ & Caractère normatif & Godfrey, hatch. 2007 \\
\hline
\end{tabular}

Source: Synthèse de l'auteur

Si l'émergence de la RSE est due à l'américain Bowen en 1953 qui postulait le caractère volontaire de l'intégration des dimensions sociales (Capron et Quairel-Lanoizelée, 2007), on devait attendre en Europe les années 90 pour passer à une démystification de la notion en faisant référence traditionnellement au concept de développement durable (Ivanaj et Mc Intyre, 2006) et en institutionnalisant un caractère obligatoire d'intégration des facteurs sociaux (Le livre vert de l’UE, CCE 2001, Loi NRE en France 2002).

Les deux approches relèvent en réalité des différences quant aux origines et aux mobiles de leur incorporation dans l'entreprise. Dans le moment où l'approche américaine (approche anglo-saxonne) puise ses fondements dans des percepts religieux et éthiques centré sur l'individu (Businessman), la RSE européenne s'inscrit plutôt dans une longue tradition de capitalisme social. Passant d'abord par d'autres concepts tels que la citoyenneté de l'entreprise ou l'approche par les parties prenantes, elle a regagné l'intérêt en Europe dans le sens d'anticipation et de prévention des risques, tandis qu'aux Etats Unis on se concentrait beaucoup plus sur la correction des effets issus des activités économiques (Capron 2003).

De manière générale, si la RSE est devenue beaucoup plus institutionnalisé en Europe, elle a par contre gardé un aspect moral et éthique dans sa portée aux Etats Unis.

Matieu 2008, quant à lui, ne perçoit pas qu'il s'agit de deux approches concurrentielles, mais plutôt comme le point de rencontre entre les deux courants exigé par la croissance de la demande de développement des 
pratiques gestionnaires sous des modèles représentant au mieux les attentes sociétales à l'égard des entreprises.

\section{Le retour de la RSE: quelle relation avec la gestion des RPS?}

L'approche libérale sous son modèle "Business case" admet la RSE dans sa stratégie tant que cette dernière contribuera positivement à la profitabilité et limitera les risques de mauvaise gouvernance. (Dyllick et Hockerts, 2002, cité par Benyyder 2009). Selon eux la RSE devient un facteur de compétitivité de l'entreprise dans la mesure où le bien être social et la performance environnementale permettent d'améliorer l'efficacité et l'efficience économique de l'entreprise.

La perception de l'entreprise vis à vis de ses parties prenantes (Igalens et Tahri 2012) dépend du pouvoir qu'elles détiennent, de leur légitimité, et de l'urgence à traiter leur problème (Mitchell et al., 1997). La particularité des salariés c'est qu'ils font partie intégrante de l'entreprise et exercent un pouvoir important puisqu'ils sont à l'origine de la performance de l'organisation ou au contraire être source de problèmes. L'apparition des RPS revient à diminuer la performance et le bien être au travail contribuant ainsi à la dégradation de la motivation des salariés (collaborateurs) comme présenté en ce qui suit:

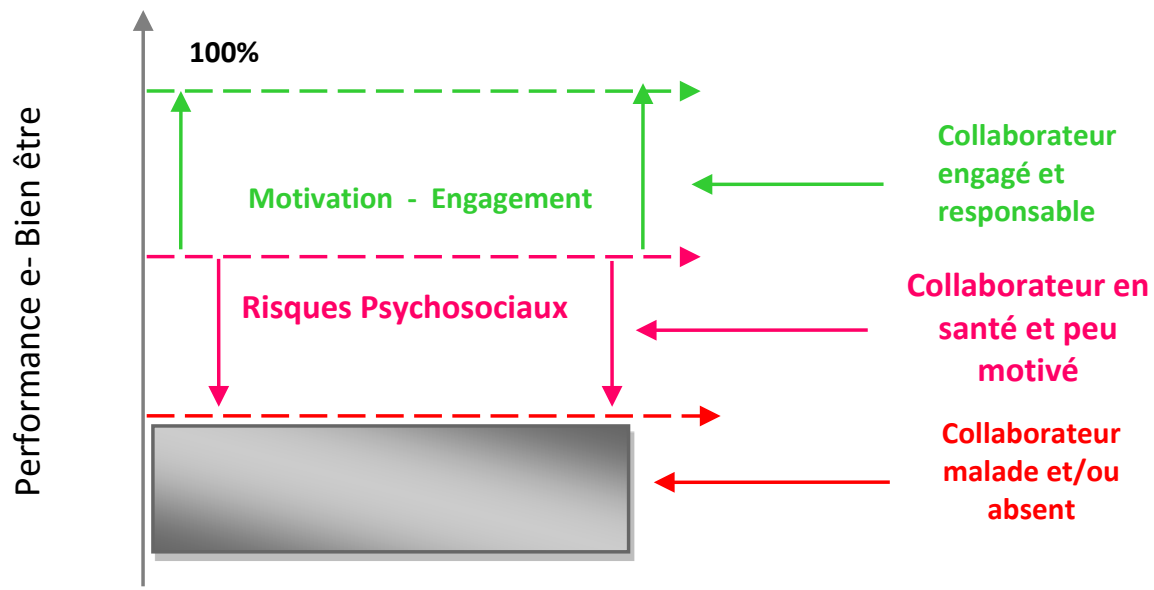

Figure 1: Impact des RPS sur la motivation des collaborateurs.

Analyser l'impact de la RSE sur le bien être des salariés revient à comprendre à travers le contrat psychologique les mécanismes d'intégration de la RSE dans la gestion des ressources humaines par la réconciliation entre l'économique et le social. Ainsi, le bien être au travail constitue une variable explicative capitale de la performance sociale qui ne pourrait pas être explicitée sans un bon niveau de maîtrise des RPS.

D'ailleurs, sous une logique de développement durable qui place l'homme au cœur des préoccupations de l'entreprise (Leroux et Van de 
Portal, 2011), la gestion des RPS fait partie importante du champ de la RSE dans la mesure où elle forme une nouvelle modalité de gestion et d'instrumentalisation des initiatives de gestion des RPS. En concert, elle représente l'occasion d'optimiser les techniques de prévention et de traitements des RPS.

\section{Modèles théoriques des RPS:}

Conçus comme une affaire de management, les modes d'organisation du travail créent parfois des différents dysfonctionnements d'ordre technique, procédural, relationnel, ayant des incidences sur les résultats des entreprises et des individus qui la composent soit au niveau de leur santé physique ou psychique.

\section{Définition des risques psychosociaux}

En réalité, ce n'est que récemment que la question des risques psychosociaux au travail a été reconnue par les entreprises sous une approche objective (Manzano 2010) du moment où ils engendrent des coûts cachés non négligeables qui affectent la performance de l'entreprise et la société en même temps. D'ailleurs plusieurs études-actions ont traité cette question de la détermination de la place des modes d'organisation de travail dans la génération des RPS. (Degré de responsabilisation des entreprises par ARACT Martinique 2009) et ont conclu que certes les modes d'organisation de travail agréent la création des facteurs de RPS, mais ils ne sont pas responsables de toutes les tentions sociales qui affectent la santé psychique des salariés.

La particularité de la question des RPS provient de sa large multidisciplinarité théorique partant des sciences humaines, psychologie du travail, ergonomie, sociologie, sciences biomédicales, médecine du travail, et sciences économiques. Dans ce domaine, où vie privée et vie professionnelle se confondent, les RPS révèlent des dimensions subjectives et des aspects multifactoriels qui en font un phénomène difficile à identifier et à définir (EUROGIP 2010).

Les praticiens de la gestion et la prévention des RPS reconnaissent leur apparition lorsqu'il y a un déséquilibre dans le système constitué par l'individu et son environnement de travail. Il s'agit d'une probabilité d'apparition du trouble psychosocial ayant pour origine l'environnement professionnel.

Plusieurs sont les études et les enquêtes européennes qui avaient pour vocation la projection des RPS et leur classement par facteur de risque (Etude INRS 2013 et 2014, EU-OSHA 2013, Réseau ANACT 2008, enquête Sumer 2003, etc. ...). Nous retenons dans ce travail les RPS les plus fréquents et les plus critiques dans le système organisationnel à savoir : le 
stress au travail, la souffrance au travail, les violences au travail, les harcèlements (moral et sexuel), les conditions addictives du travail, l'épuisement professionnel. En ce qui suit nous présentons brièvement ces RPS.

Commençons d'abord par la définition du stress qui désigne une situation où il y a déséquilibre entre la perception qu'a une personne à des contraintes que lui impose son environnement et la perception qu'il a de ses propres ressources pour y faire face. (ARACT 2009). Il affecte non seulement les qualités psychologiques mais aussi la santé physique, le bien être, et la performance de la personne. Bien que le ressenti du stress reste individuel, ses déterminants et ses effets sont d'ordre individuel et collectif à la fois et sont fortement liés aux conditions de travail.

En ce qui concerne la souffrance au travail, nous admettons que les modes d'organisation qui nient ou écartent systématiquement l'individu, son identité, sa propre conception de travail, ses valeurs et ses espoirs constituent des sources importantes de la souffrance au travail. Ces dernières (liées au vécu ou à l'organisation du travail) sont souvent en interaction et/ ou en interpénétration.

Quant aux violences au travail (agression, domination, persécution), elles se divisent en violences internes et externes et dégagent des liens de causalité avec le stress de manière à ce que le stress peut être la conséquence d'une violence et inversement peut engendrer une violence ou une souffrance. (voir figure). Il est à noter aussi que les violences comportent encore les harcèlements moral et sexuel déterminés comme l'ensemble des conduites abusives gestes, paroles, comportements, et attitudes qui portent atteinte, par leur répétition ou leur systématisation, à la dignité ou à l'intégrité psychique ou physique d'une personne, mettant en péril l'emploi de celle-ci ou dégradant le climat de travail. D'un autre côté, et en plus des comportements d'harcèlement, les conduites addictives (comportements d'accoutumance et de dépendance) créent également des liens de causalité avec le stress, les violences ou la souffrance au travail.

En dernier, l'épuisement professionnel comporte essentiellement " l'épuisement physique et émotionnel qui conduit au développement d'une image de soi inadéquate, d'attitudes négatives au travail ave perte d'intérêt de ce que l'on fait" (ARACT 2009).

L'ensemble de ces définitions souscrivent des liens de causalité complexes existants entre les RPS et leur facteurs, de manière à ce que les mêmes facteurs organisationnels ou psychologiques constituent la source des différents autres troubles sous forme d'un cercle vicieux (ARACT Martinique 2009) comme présenté sur le schéma: 


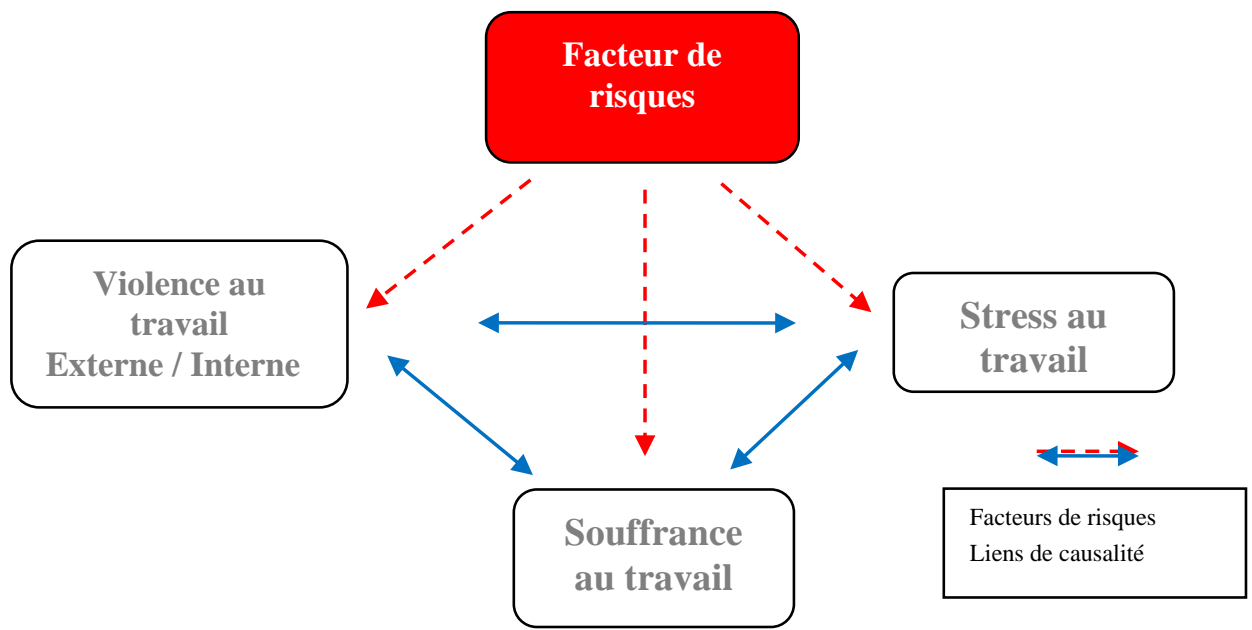

Figure 2: Liens de causalité entre facteurs de risques et risques psychologiques. Source: ARACT 2009.

La littérature a fait émergé depuis les années 80 deux modèles théoriques explicitant les liens existants entre les phénomènes psychosociaux au travail et le développement de certaines maladies.

Le modèle KARASEK qui permet de situer les salariés sur un graphique défini par deux axes : la demande psychologique en ordonnée et la latitude décisionnelle en abscisse et observe qu'il y a un risque d'avoir des répercussions négatives sur la santé des salariés lorsqu'ils ont à la fois une demande psychologique relativement élevée et une latitude décisionnel relativement faible (Wassenhove 2014). (C'est ce que le modèle Karasek appelle job strain ou de tension au travail). Karasek souligne également que d'autres caractéristiques organisationnelles peuvent modérer ces effets négatifs sur un salarié à savoir le soutien social dans ses différentes dimensions tangible, instrumentale, informationnelle et émotionnelle. Ce concept de soutien social a été ajouté au modèle vers la fin des années 80 (Jonshon \& Hall, 1988, Jonhson \& al,. 1989, Jonhson \& al,. 1996 cité par Conne-Perréard \& al., 2001).

Le modèle du Déséquilibre Efforts/Récompenses (Niedhammer et Siegrist 1998 cité par Conne-Perréard \& al., 2001) explique quant à lui que la situation de travail caractérisée par des efforts élevés et des récompenses faibles s'accompagne souvent par des réactions pathologiques sur les plans émotionnel et physiologique. 


\section{Les facteurs organisationnels générateurs des RPS}

L'apparition et le développement des RPS naissent essentiellement des rapports d'interaction et de dépendance existants entre les salariés et leur organisation. En concret, ces rapports se structurent dans une situation d'équilibre de manière à ce que les salariés s'investissent pour répondre aux objectifs et exigences de leur organisation et en contre partie, cette dernière met tout les moyens nécessaires à leur disposition pour la réalisation des ses objectifs. La divergence entre leur objectifs créent un climat favorable à l'apparition des RPS marqué par des sources potentiels de tensions qui sont déjà en interaction. (Ndjaboué \& al., 2012).

Le système de régulation permet en réalité une adaptation permanente entre les objectifs et les moyens de l'organisation et ceux de l'opérateur, chose qui réalise l'équilibre entre les différentes composantes du travail et les caractéristiques individuelles du salarié. En pratique, Lorsque les tensions ne sont pas ou sont mal régulées, chacune des composantes du travail peut devenir un facteur à l'origine de risques psychosociaux comme illustré sur la figure suivante. (ARACT 2009).

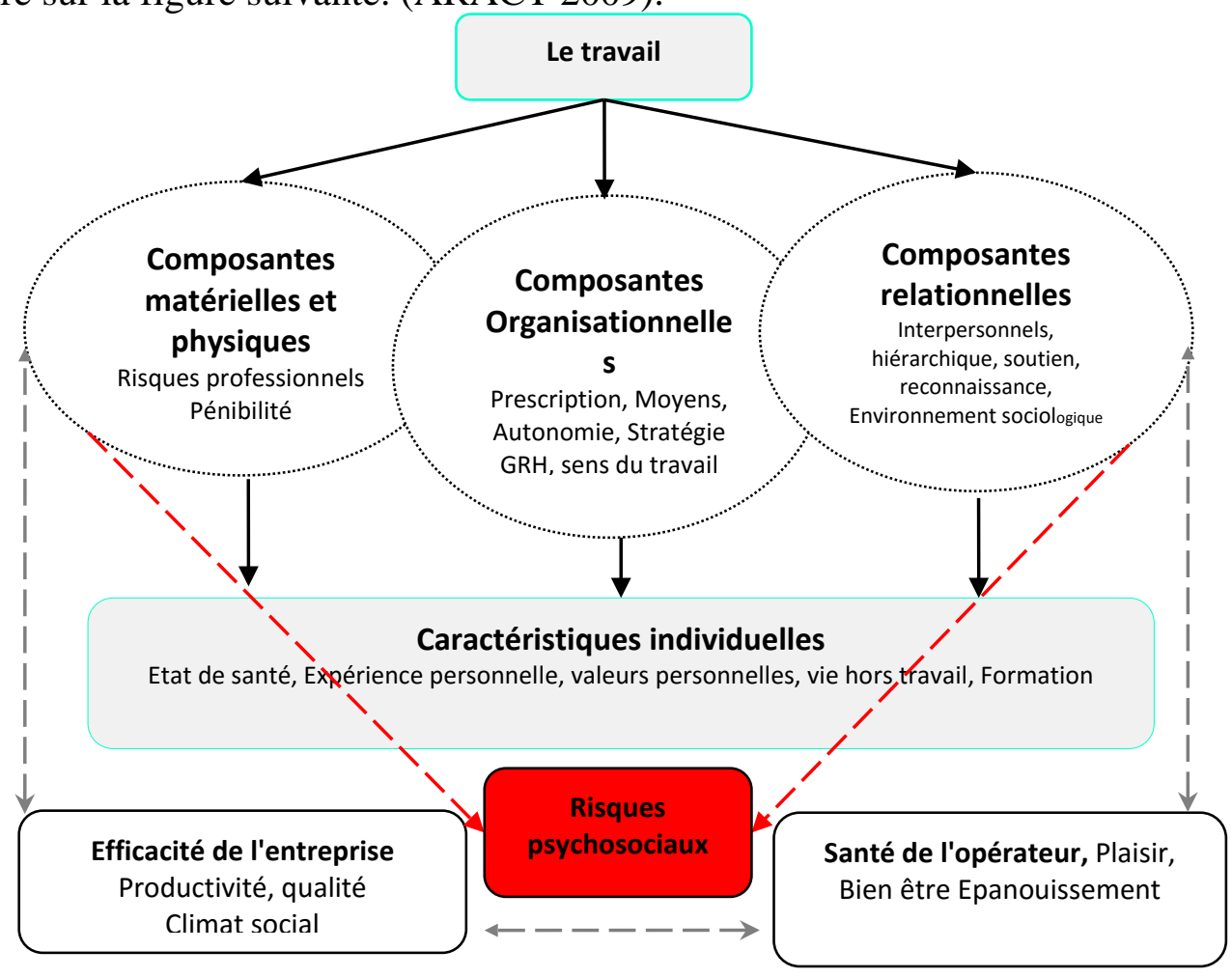

Figure 3: Système de travail où les tensions ne sont pas régulés.

Source: ARACT 2009. 
Afin de comprendre l'émergence de ces RPS, il devient indispensable de comprendre les causes et les facteurs des troubles psychosociaux que les praticiens en gestion de ressources humaines ont résumé dans les points suivants (Technologia 2013) en plus d'un contexte économique, social et environnemental beaucoup plus complexe et plus compétitif, marqué par la mondialisation, la crise et la peur du chômage:

\section{Le développement du « Lean management "}

Plusieurs chercheurs (CEE, 2006) ont démontré que le lean management est un mode d'organisation générateur de stress et aggravant les risques de dégradation des conditions de travail et d'atteintes à la santé mentale. Cette dégradation est due en concret au fonctionnement du lean management qui se base sur l'identification et l'élimination de toutes les fromes de gaspillage, de toute les sources d'inefficacité et de non valeur ajoutée (automatisation systémique nuisible à l'être humain).

\section{L’individualisation des méthodes managériales}

Les pratiques managériales mettant en concurrence permanente les salariés en privilégiant la compétition sur la coopération (benchmark) affectent sévèrement la santé mentale des salariés et mettent en échec les stratégies de défense collective face aux contraintes du travail et renforcent l'isolement au travail (Montreuil 2014).

Dans l'entreprise, l'évolution des modes de management pose aussi la question fondamentale de l'exercice du pouvoir dans l'entreprise et la faiblesse du dialogue social ( l'expression des salariés sur leurs conditions de travail), ce qui prend plusieurs formes de perturbations comme les retards, les malfaçons, stress, absentéisme etc..

Le bien être au travail, la sécurité peuvent être aussi affectés par les différentes restructurations et changements organisationnels conduits (exemple la mobilité subie) sans anticipation de leur impact potentiel sur la santé, d'un côté et par la mauvaise conception et configuration des lieux de travail (ambiances de travail, bruit, température, lumière, TIC et techniques de géo localisation etc. ..) (IRES 2012).

Ainsi les différents facteurs sources d'émergence potentielle des RPS peuvent commencer de l'intensité excessive de travail ainsi que la complexité élevée des exigences et les contraintes au travail, qui peuvent générer des atteintes physiques et morales via le stress chronique et les accidents de travail. (exemple des objectifs moins clairs, non adéquats avec les moyens, non compatibles avec les instructions, improvisation, interruption, vigilance permanente...) .

Les horaires de travail difficiles peuvent aussi affecter la santé physique et mentale des salariés, en raison des interférences qu'elle peuvent 
occasionner avec les engagements familiaux ou sociaux ce qui rend également difficile la conciliation entre la vie professionnelles et la vie personnelle (exemple des horaires atypiques, extension des disponibilités etc. ..). En plus, l'exposition des salariés à des situations de tensions épuisantes psychologiquement peut être également éprouvante sur le plan émotionnel et exige une charge mentale et psychologique forte dans la réalisation du travail.

La faible ou l'insuffisance autonomie au travail traduite par une faible manœuvre accordée aux salariés par l'organisation représente un risque cardiovasculaire et des risques pour la santé mentale, en plus elle tend à appauvrir l'intérêt du travail et à dévaloriser les salariés et leurs compétences professionnelles (exemple de l'autonomie dans les tâches, interruption au moment du travail etc....).

La qualité des rapports sociaux au travail et les relations interpersonnelles entre salariés et entre salariés et organisation a une grande influence sur la santé physique et mentale des salariés (intégration des nouveaux, soutien des supérieurs hiérarchiques, valorisation des compétences, courtoisie et respect mutuel).

Un autre facteur qui peut générer des RPS est évoqué par l'ensemble des conflits de valeurs intrapsychiques dans l'organisation ajouté à l'insécurité de l'emploi peuvent réduire le sentiment de maîtrise de situation (exemple: insécurité socioéconomique, changement non maîtrisé de la tâche et des conditions de travail: incertitude sur l'avenir de son métier, peur de devoir changer de qualification ou de métier ...).

\section{Méthodologie de la RSE vis à vis des RPS}

La démarche RSE s'appuie sur une approche objective et collective (améliorer le bien être collectif) des RPS dus au travail, et porte ses réflexions au niveau de la ré interrogation des modalités d'exécution et d'organisation du travail, elle cherche à apprécier la qualité des relations au travail, et à repérer les zones de dysfonctionnements pour améliorer les pratiques managériales. (Conne-Perréard \& al., 2001)

La RSE s'appuie sur l'approche des parties prenantes dont les salariés en constituent une composante principale. Le bien être de l'individu est un objectif stratégique pour la démarche RSE. Conçue sous forme bonnes pratiques (Tahri, 2010), la RSE se propose d'assurer l'employabilité des salariés grâce à la formation, leur permettre de s'épanouir en veillant au bon équilibre entre vie professionnelle et privée, assurer l'égalité des chances entre hommes et femmes, respecter la diversité etc... 


\section{Les actions de la RSE sur les RPS}

- L'instauration de la démarche RSE dans l'entreprise permet d'intégrer la santé au travail comme une composante de la stratégie globale de l'entreprise au plus haut niveau de gouvernance. Les managers sont appelés à formuler un regard stratégique d'anticipation en plaçant l'homme au centre de l'entreprise pour redonner au travail sa valeur d'intégration et d'émancipation. La RSE permettra ainsi d'appréhender la santé et sécurité au travail en assurant la prévention et le traitement des risques psychologiques.

- La démarche RSE voit dans l'adoption d'un management par confiance une clé importante de la prévention des RPS par la promotion d'un dialogue social de qualité autour de la santé et du bien-être au travail, car en participant à l'élaboration du diagnostic des RPS les salariés devient susceptibles de participer individuellement à un processus collectif et ainsi exprimer son rapport au travail.

- Le RSE se base sur les actions de prévention s'attaquant directement aux sources d'atteinte à la santé psychologique. Pour une démarche RSE c'est promouvoir des modes d'organisation qui préservent la santé et la sécurité des salariés et traitant la question de pénibilité, de l'usure professionnelle, du maintien de l'emploi des seniors, de la chronicité des maladies, de la conception et l'aménagement des locaux de travail, de l'articulation entre vie professionnelle et vie privée.

- La RSE s'intéresse aussi au rôle de la formation initiale et continue des acteurs de l'entreprise pour les aider à repérer les situations à risque et élaborer des plans d'actions.

- La RSE consiste pour les entreprises à réaliser le bilan social pour recueillir les données quantitatives descriptives permettant d'avoir une vision précise sur l'intensité du travail, la santé du personnel ou la qualité des relations sociales. Un bilan social au sens de RSE doit évoluer pour en faire un rapport annuel de performance sociale composé d'un panel d'indicateurs sociaux mesurant l'impact social. 


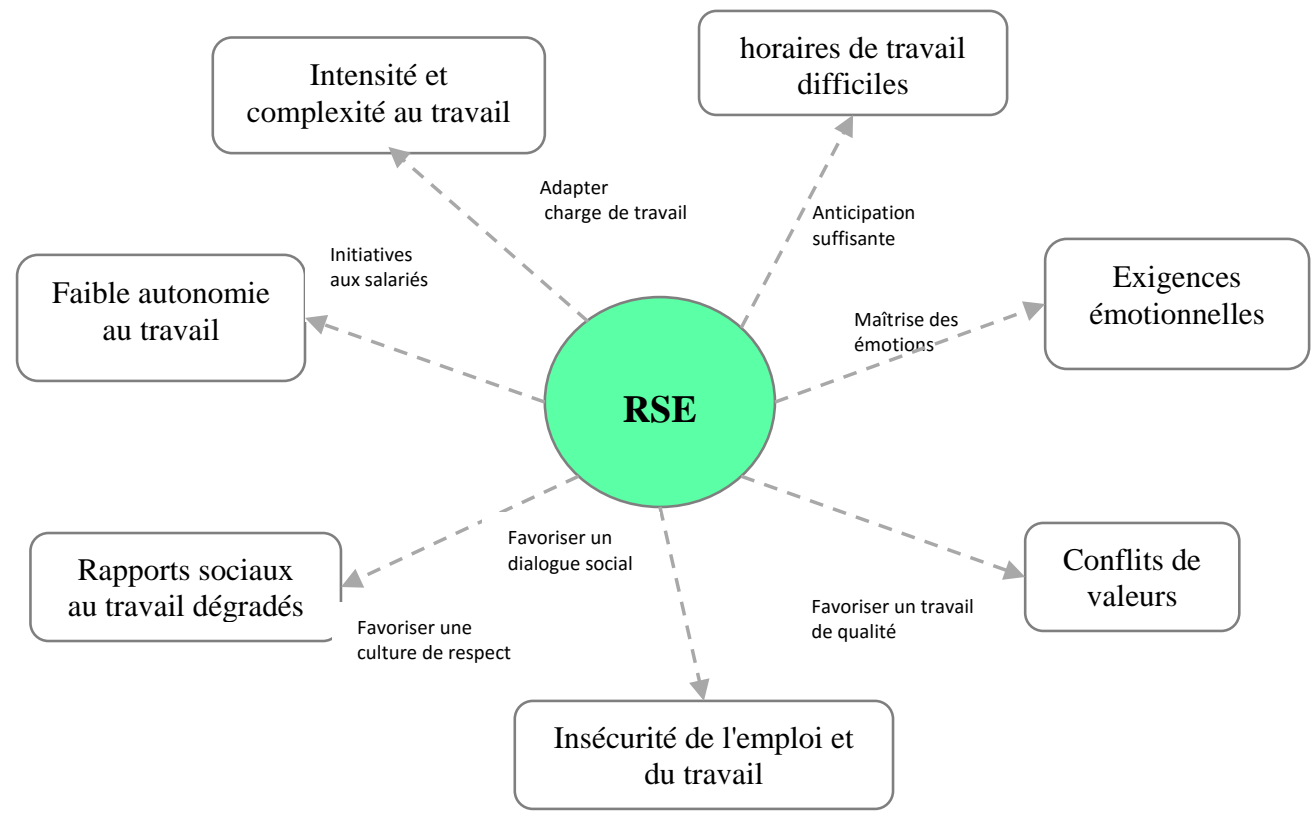

Figure 4 : Action de la RSE sur les facteurs des risques RPS

Source: Synthèse de l'auteur

La capacité de la RSE à agir sur les facteurs des RPS provient en fait de sa vocation à constituer les conditions favorables au bien être au travail comme détaillé sur le tableau suivant:

Tableau 2: Action de la RSE sur les facteurs des RPS

\begin{tabular}{|c|c|}
\hline Facteurs de RPS & Actions RSE \\
\hline $\begin{array}{l}\text { Contraintes de } \\
\text { rythme de travail }\end{array}$ & $\begin{array}{l}\text { - Prendre en compte la variabilité entre les salariés en ce qui concerne les } \\
\text { cadences et les rythmes de travail } \\
\text { - Fixer la charge de travail ou les objectifs de rendement } \\
\text { - Prendre en compte le temps d'apprentissage (nouvel recruté, changement de } \\
\text { poste) }\end{array}$ \\
\hline $\begin{array}{l}\text { Niveau de précision } \\
\text { des objectifs de } \\
\text { travail , leur } \\
\text { adéquation et } \\
\text { compatibilité entre } \\
\text { eux. }\end{array}$ & $\begin{array}{c}\text { Formaliser clairement le contenu des fonctions, mise à jour régulière des } \\
\text { instructions du travail pour tenir compte des évolutions de la réalité du travail } \\
\text { et vérifier la cohérence entre elles. } \\
\text { S'assurer que chaque salarié a une vision claire sur les priorités. } \\
\text { Prévoir des rencontres régulières pour discuter des moyens et des échéances et } \\
\text { des difficultés } \\
\text { Prendre en compte la possibilité des aléas et imprévus et anticiper le recours à } \\
\text { des ressources supplémentaires. }\end{array}$ \\
\hline $\begin{array}{l}\text { Gestion de la } \\
\text { polyvalence }\end{array}$ & $\begin{array}{c}\text { Planifier la rotation des postes } \\
\text { Former le personnel aux divers postes qu'il est susceptible d'occuper. }\end{array}$ \\
\hline $\begin{array}{l}\text { Interruption dans le } \\
\text { travail }\end{array}$ & $\begin{array}{c}\text { Analyser la nature des diverses interruptions : identifier celles qui sont } \\
\text { inévitables et celles qui peuvent être limités, différées }\end{array}$ \\
\hline $\begin{array}{c}\text { Attention et } \\
\text { vigilance dans le } \\
\text { travail }\end{array}$ & $\begin{array}{l}\text { Adapter la durée de vacation pour les postes avec de fortes exigences } \\
\text { d'attention et de vigilance afin d'éviter la baisse de performance et la fatigue. } \\
\text { Permettre aux salariés de prendre des pauses quand ils souhaitent } \\
\text { Alterner les tâches pour éviter les effets de monotonie et d'ennui }\end{array}$ \\
\hline
\end{tabular}




\begin{tabular}{|c|c|}
\hline $\begin{array}{l}\text { Durée } \\
\text { hebdomadaires du } \\
\text { travail }\end{array}$ & $\begin{array}{c}\text { Limiter autant que possible le recours aux heures supplémentaire. Si non: en } \\
\text { établir une programmation et donner un préavis compatibles avec les } \\
\text { contraintes personnelles } \\
\text { Transparence et paiement des heures supplémentaire. }\end{array}$ \\
\hline $\begin{array}{l}\text { Travail en horaire } \\
\text { atypique }\end{array}$ & $\begin{array}{l}\text { Limiter le recours au travail de nuit } \\
\text { Limiter l'exposition aux horaires atypiques. } \\
\text { Rendre possible des pauses en fonction de l'état de fatigue. }\end{array}$ \\
\hline $\begin{array}{l}\text { Extension de la } \\
\text { disponibilité }\end{array}$ & Limiter les sollicitations professionnelles \\
\hline $\begin{array}{l}\text { Prévisibilité des } \\
\text { horaires de travail et } \\
\text { anticipation des } \\
\text { changements }\end{array}$ & $\begin{array}{c}\text { Planifier le travail suffisamment à l'avance } \\
\text { Définir les conditions et les délais de prévenance des changements de } \\
\text { planning. }\end{array}$ \\
\hline $\begin{array}{l}\text { Conciliation entre } \\
\text { vie privée et vie } \\
\text { professionnelle }\end{array}$ & $\begin{array}{c}\text { Aider la salariés à la reprise des horaires normaux en cas de contraintes } \\
\text { personnelles } \\
\text { Adapter les horaires de travail au mieux pour les rendre compatibles avec la } \\
\text { vie familiale et sociale. } \\
\text { Prendre en compte les horaires du transport. }\end{array}$ \\
\hline $\begin{array}{l}\text { Tensions avec le } \\
\text { public }\end{array}$ & $\begin{array}{c}\text { Définir une politique d'entreprise envers les incivilités et violences. } \\
\text { Mettre ne place des procédures de protection adaptées aux situation de travail } \\
\text { Former les salariés à des situations de violence externe }\end{array}$ \\
\hline $\begin{array}{l}\text { Confrontation à la } \\
\text { souffrance d'autrui }\end{array}$ & Mettre les salariés en équipes pour délibérer des situations difficiles \\
\hline $\begin{array}{l}\text { Maîtrise des } \\
\text { émotions }\end{array}$ & $\begin{array}{c}\text { Prendre en compte la variabilité entre les salariés en ce qui concerne les } \\
\text { cadences et les rythmes de travail } \\
\text { Fixer la charge de travail ou les objectifs de rendement } \\
\text { Prendre en compte le temps d'apprentissage }\end{array}$ \\
\hline $\begin{array}{l}\text { Autonomie dans la } \\
\text { tâche }\end{array}$ & $\begin{array}{c}\text { Laisser des marges de manœuvre aux salariés dans la manière d'atteindre leur } \\
\text { objectif et permettre des prises d'initiative de la part des salariés }\end{array}$ \\
\hline $\begin{array}{l}\text { Autonomie } \\
\text { temporelle }\end{array}$ & $\begin{array}{c}\text { Donner la possibilité de choisir les moments de pause, prévoir et aménager des } \\
\text { espaces de détentes. }\end{array}$ \\
\hline $\begin{array}{l}\text { Utilisation et } \\
\text { développement des } \\
\text { compétences }\end{array}$ & $\begin{array}{l}\text { Mettre en place un plan de formation en adéquation avec les besoins de } \\
\text { l'entreprise , le profil et les attentes des salariés. }\end{array}$ \\
\hline $\begin{array}{l}\text { Soutien de la part } \\
\text { des collègues }\end{array}$ & $\begin{array}{c}\text { Eviter les postes de travail isolé , faciliter les pauses collectifs et les moments } \\
\text { de convivialité. }\end{array}$ \\
\hline $\begin{array}{l}\text { Soutien des sup } \\
\text { hiérarchiques }\end{array}$ & $\begin{array}{c}\text { Encourager le dialogue, l'écoute, la confiance, la transparence, l'équité, la } \\
\text { reconnaissance , la politesses et les mises à l'écart } \\
\text { Former l'encadrement de proximité au management des équipes et à la } \\
\text { résolution des conflits. }\end{array}$ \\
\hline $\begin{array}{c}\text { Violence interne au } \\
\text { travail }\end{array}$ & Bannir la violence et favoriser une culture de respect \\
\hline $\begin{array}{l}\text { Reconnaissance au } \\
\text { travail }\end{array}$ & Valoriser en interne les différents métiers \\
\hline Qualité empêchée & $\begin{array}{c}\text { Anticiper sur les délais et la charge de travail, prévoir les moyens matériels et } \\
\text { humains compatibles avec un travail de qualité }\end{array}$ \\
\hline Travail inutile & Examiner attentivement les tâches signalées comme inutiles \\
\hline $\begin{array}{c}\text { Insécurité } \\
\text { socioéconomique }\end{array}$ & $\begin{array}{c}\text { Instaurer une communication interne et transparente sur les évolutions et les } \\
\text { changements. }\end{array}$ \\
\hline $\begin{array}{l}\text { Conduite du } \\
\text { changement dans } \\
\text { l'entreprise }\end{array}$ & $\begin{array}{c}\text { Communiquer avec les salariés pour éviter les risques d'interprétation ou de } \\
\text { déformation de l'information, des rumeurs } \\
\text { Former aux nouveaux procédés. }\end{array}$ \\
\hline
\end{tabular}


L'étude du retour de la RSE reste une question encore ambigüe et difficile à maîtriser (Igalens \& Gond 2003). En réalité, nous cherchons à comprendre les effets positifs du déploiement de la RSE sur les changements sociaux et à analyser son impact sur la criticité des RPS.

Ainsi nous allons analyser les questionnaires administrés au niveau de l'ANP sur trois volets: le premier porte sur le déploiement de la RSE de manière générale, puis un deuxième qui évalue le volet social du déploiement de la démarché RSE et en fin un troisième questionnaire qui a porté sur le point de vue de 25 collaborateurs sur un total de 93 avec $20 \%$ d'encadrement sur leur perception des RPS, sur le niveau de leur bien être au travail et sur les changements induits par la démarche RSE.

\section{La RSE comme outil de gestion des RPS dans l'ANP}

Le transport maritime connaît sous les pressions de la globalisation de l'économie de grands changements tels que l'augmentation de la taille des navires, la fusion des grands transporteurs maritimes, le rôle capital des ports dans les chaînes logistiques et l'émergence de grands opérateurs de terminaux portuaires. C'est ainsi qu'aujourd'hui, une grande pression est exercée sur les ports pour adapter leurs capacités et fournir des services performants. De tels défis exigent une pression plus accrue sur les collaborateurs ce qui crée des conditions favorables à l'émergence des RPS.

Dans sa vocation à contribuer au développement économique et social au niveau national et régional au bénéfice de tous les stakholders, l'ANP concrétise sa contribution par la création de la valeur pour les opérateurs économiques et les citoyens, dans un environnement responsable et durable à travers une démarche évolutive de responsabilité sociale qui préserve l'avenir.

Les changements organisationnels impliqués par l'intégration d'une politique RSE se concrétisent au niveau d'un système de management proactif et évolutif qui intègrent les initiatives et les apprentissages orientés RSE, et permettent la mesure de l'impact social. En effet, l'efficacité de l'engagement dans la RSE dépend largement de ses pratiques managériales d'un côté et de l'attitude de ses collaborateurs qui doivent approprier leur comportements et leurs contributions possibles. (Rambaud \& al,. 2009). Igalens, Tahri (2012), distinguent les perceptions de la RSE basées sur les parties prenantes et sur le développement durable et affirment qu'aucune perception n'a été consacrée aux salariés.

Ainsi, Notre investigation empirique chez l'ANP sera articulée autour de trois points discutant de notre problématique: d'abord nous présentant l'état des lieux du déploiement et de la maturité de la RSE chez l'ANP à travers le test de matérialité, puis nous présentons une évaluation du volet social de la démarche à travers la méthode des scores, puis en fin nous 
comparons entre les niveaux de criticité des RPS avant et après le déploiement de la démarche de RSE depuis 2014.

\section{Etat des lieux : La RSE constitue un défi stratégique pour l'ANP.}

La politique portuaire marocaine répond à la fois à des exigences économiques et politiques. D'abord, elle répond à une demande portuaire exprimée par les partenaires économiques nationaux et les acteurs des transports internationaux et puis elle contribue aux objectifs politiques nationaux qui portent sur la valorisation des avantages comparatifs du pays vis-à-vis de son contexte méditerranéen et international. La politique portuaire vise également à garder les équilibres régionaux du Royaume et favoriser le développement social et humain tout en soutenant les dynamiques économiques.

Pour cela la démarche RSE est perçue par l'ANP comme une conviction menée dans une perspective de respect de l'homme et de l'environnement d'une part et comme une opportunité d'adhésion et de motivation des employés.

L'approche RSE dans l'ANP a été mise en place depuis 2014 avec la collaboration des experts d'organismes externes et avec concertation des représentants du personnel. Certes, l'organisme ne dispose pas jusqu'à maintenant d'un service spécialement dédié RSE mais il s'inscrit dans des pratiques rendant compte de ses performances RSE en termes de rapport, questionnaires etc....en plus des programmes de sensibilisation pour les employés (formation, expositions, conférences ...)

Pour le pilier social, l'ANP préconise des politiques spécifiques de lutte contre les discriminations liées au sexe, et contre les discriminations liées à l'origine (ethnique, sociale et culturelle).

En plus de l'élaboration de l'inventaire des risques professionnels, l'ANP établit des mesures préventives pour pallier ces risques et elle prépare des plans d'action de prévention des risques psychosociaux (stress, mal être au travail, harcèlement etc....).

Intéressée par l'amélioration continue des compétences des ses employés, l'ANP s'engage dans des dépenses importantes pour la formation professionnelle (plus de $5 \%$ de la masse salariale) en mettant en place des mesures de gestion prévisionnelle des emplois et des compétences de manière régulière (visibilité du programme 2030).

Pour identifier le niveau de maturité RSE chez l'ANP nous avons utilisé le test de matérialité conçu sous forme d'une approche méthodologique pour identifier et hiérarchiser les enjeux RSE les plus importants. Le test s'est fait sur la base de l'analyse des facteurs externes et internes liés aux risques et opportunités ayant une influence sur l'organisation. 
L'utilité du test apparaît lorsqu'il permet à l'organisation de concentrer ses ressources sur les enjeux RSE les plus stratégiques et les plus riches en apport de valeur ajoutée d'un côté, puis il permet de rendre les politiques RSE et la stratégie opérationnelle de compétitivité compatibles.

L'originalité de l'outil préconisé par les acteurs de la RSE repose sur la double priorisation de chaque enjeu, d'une part du point de vue interne de l'entreprise (dans une optique "business") et d'autre part du point de vue externe des parties prenantes.

Ainsi, en analysant les attentes des employés et l'engagement de leur organisation dans la RSE nous avons élaboré la matrice de matérialité par les poids donnés par chaque partie aux composantes de la RSE sur son volet social comme présenté par les combinaisons sur la figure suivante:

Figure 5: Test de matérialité du volet social de la RSE dans l'ANP

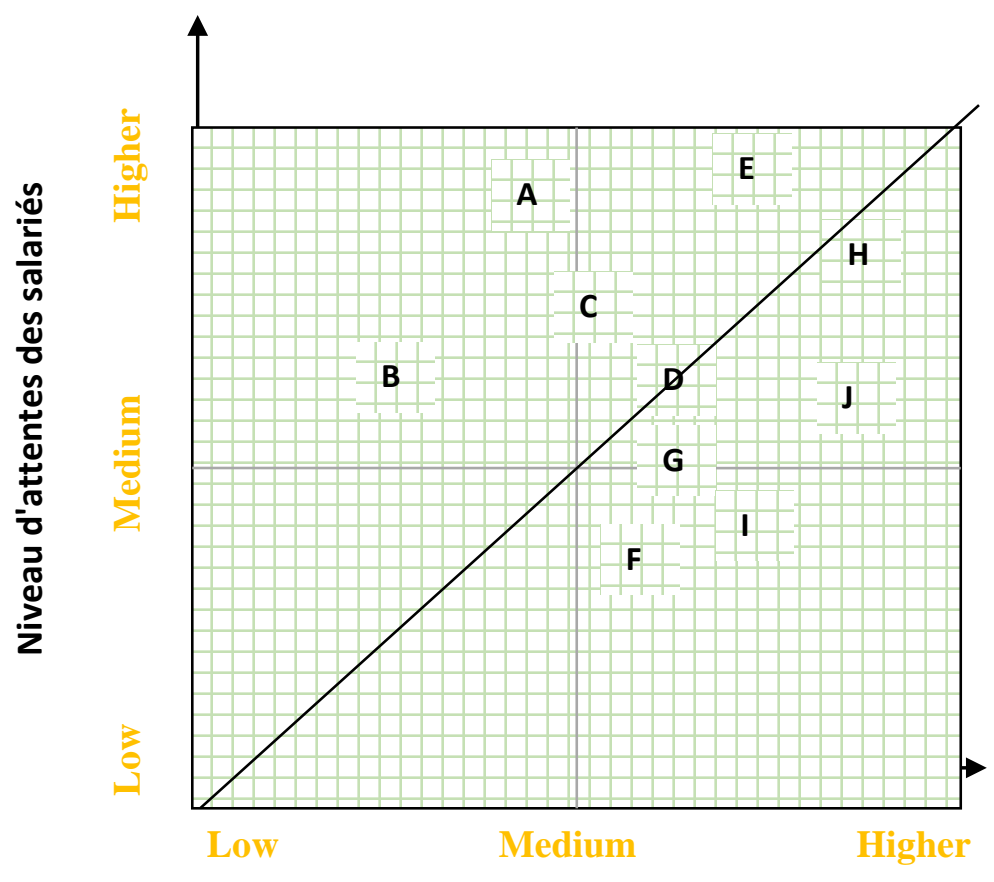

Niveau d'importance donné par l'Organisation

Tableau 3: Eléments clés ayant un poids pour l'Organisation et les employés

\begin{tabular}{|c|c|}
\hline A: Intensité et complexité au travail & F: Gestion des conflits \\
\hline B: Horaires du travail & G: Exigences émotionnelles \\
\hline C: Autonomie au travail & H: Bien être au travail \\
\hline D: Rapports sociaux & I: Compétences des employés \\
\hline E: Santé et sécurité au travail & J: Diversité et égalité \\
\hline
\end{tabular}


Les attentes des salariés de la démarche sont très élevés au niveau de trois éléments clés à savoir l'intensité et la complexité du travail, le bien être au travail et la santé et sécurité au travail. Cette dernière suppose une maîtrise des risques professionnels encours y compris la composante psychosociale.

Par contre, les supérieurs hiérarchiques responsables de la conduite et de la réussite de la RSE devient plus stressés et plus inquiets des résultats relatifs aux niveaux de satisfaction des parties prenantes de la démarche. Ce qui nous amène à poser une autre réflexion : si la RSE contribue à l'amélioration du bien être au travail des employés, est ce qu'elle ne constitue pas un facteur générateur de stress pour les dirigeants et les supérieurs hiérarchiques? Est ce qu'elle ne se fait pas à leur détriment?

\section{Evaluation de l'apport social de la RSE}

En effet, les dispositifs d'évaluation actuellement utilisés par l'ANP pour mesurer les progrès réalisés grâce à leurs démarches RSE n’apportent pas de réponses satisfaisantes puisqu'ils sont toujours en cours de formalisation et d'évaluation.

A partir de 2014, la démarche RSE a permis pour l'ANP de veiller relativement au bien être des employés, d'assurer un environnement de travail agréable et adapté, d'assurer la prévention et la gestion du stress au travail et d'autres risques psychosociaux.

Le tableau suivant décrit les différents scores donnés par l'ANP aux actions de la RSE selon les domaines:

Tableau 4 : Scores de RSE selon les domaines

\begin{tabular}{|c|c|c|c|c|}
\hline \multirow{2}{*}{\multicolumn{3}{|c|}{\begin{tabular}{|l|l|} 
Nule : 0 & Faible: 1 \\
Domaine Responsabilité sociale \\
\end{tabular}}} & \multicolumn{2}{|c|}{ Très fort : 4} \\
\hline & & & Score & \multirow[t]{2}{*}{ Moyenne } \\
\hline \multicolumn{4}{|c|}{ Responsabilité dans le domaine social Score: 0.2} & \\
\hline & \multicolumn{2}{|c|}{ Favoriser le bien être et la qualité de vie au travail } & 3 & 0.075 \\
\hline & \multicolumn{2}{|c|}{ Développer les compétences de tous les salariés } & 3 & 0.075 \\
\hline & \multicolumn{2}{|c|}{ Promouvoir la diversité et l'égalité des chances } & 2 & 0.05 \\
\hline & \multicolumn{4}{|c|}{ Responsabilité dans le domaine environnemental score: 1.075} \\
\hline & \multicolumn{2}{|c|}{$\begin{array}{l}\text { Maîtriser les consommations d'énergie, d'eau et de matières } \\
\text { premières }\end{array}$} & 1 & 0.025 \\
\hline & \multicolumn{2}{|c|}{ Réduire les émissions dans l'air, les sols et l'eau } & 3 & 0.075 \\
\hline & \multicolumn{2}{|c|}{ Favoriser la réduction et la valorisation des déchets } & 1 & 0.025 \\
\hline & \multicolumn{2}{|c|}{ Garantir la pérennité de l'entreprise et sa performance économique } & 2 & 0.05 \\
\hline & \multicolumn{2}{|c|}{ Responsabilité dans le domaine économique score: 0.2} & & \\
\hline & \multicolumn{2}{|c|}{ Respecter les intérêts des clients et des consommateurs } & 4 & 0.1 \\
\hline & \multicolumn{2}{|c|}{$\begin{array}{l}\text { Établir des relations durables avec les sous-traitants et les } \\
\text { fournisseurs et respecter leurs intérêts }\end{array}$} & 4 & 0.1 \\
\hline \multirow{4}{*}{$\sum_{4}^{2}$} & \multicolumn{2}{|c|}{ Responsabilité dans le domaine territorial score: 0.25} & & \\
\hline & \multicolumn{2}{|c|}{ Contribuer au développement économique et social des territoires } & 4 & 0.1 \\
\hline & \multicolumn{2}{|c|}{ Privilégier les achats auprès de fournisseurs locaux } & 4 & 0.1 \\
\hline & \multicolumn{2}{|c|}{ Agir sur les freins à l'emploi et favoriser l'insertion } & 2 & 0.05 \\
\hline
\end{tabular}




\begin{tabular}{|l|l|l|l|}
\hline Responsabilité dans le domaine de la gouvernance score: 0.175 & & \\
\hline $\begin{array}{l}\text { S’inscrire dans une démarche d'amélioration continue et } \\
\text { d'innovation pour la RSE }\end{array}$ & $\mathbf{2}$ & 0.05 \\
\hline $\begin{array}{l}\text { Rendre des comptes de manière transparente sur les objectifs, } \\
\text { pratiques et résultats en matière de RSE }\end{array}$ & $\mathbf{2}$ & 0.05 \\
\hline $\begin{array}{l}\text { Développer un dialogue social avec les salariés et leurs } \\
\text { représentants sur la mise en } \\
\text { œuvre de ce référentiel régional }\end{array}$ & $\mathbf{3}$ & 0.075 \\
\hline
\end{tabular}

Les score les plus forts sont affectés dans la l'ANP au domaine territorial (score 0.25 ) et au domaine économique et social (même score 0.2)

Le climat social dans l'ANP est jugé par les employés moyennement serein (84\% des interrogés), dans lequel la parité homme femme est relativement respectée et les conditions de travail, hygiène et sécurité sont très bonnes.

Les RPS les plus cités par les employés sont le stress (92\%) et le stress et l'épuisement professionnel 51\%). La démarche RSE permet de les prévenir de manière partielle. Selon les responsables des ressources humaines, la vocation RSE est beaucoup plus axée sur la prévention des risques plus que la correction des effets.

Le tableau suivant décrit des exemples des actions de la RSE avant, pendant et après les RPS

\begin{tabular}{|c|c|}
\hline \multirow{2}{*}{ RPS } & Tableau 5: Action de la démarche RSE \\
\hline & Avant le risque (préventive) \\
\hline Stress & Exemple : Départ en congés, améliorer les contraintes au rythme du travail, \\
\hline Epuisement & Exemple : Consulter le Médecin au travail \\
\hline \multicolumn{2}{|r|}{$\begin{array}{c}\text { Pendant le risque } \\
\end{array}$} \\
\hline Stress & $\begin{array}{l}\text { Exemple: Faire des arbitrages et des choix , augmenter 'autonomie dans les } \\
\text { tâches }\end{array}$ \\
\hline Epuisement & $\begin{array}{c}\text { Exemple: Redéploiement si nécessaire, limiter ou éliminer les horaires } \\
\text { atypiques }\end{array}$ \\
\hline \multicolumn{2}{|r|}{$\begin{array}{c}\text { Après le risque } \\
\end{array}$} \\
\hline Stress & Exemple: Modulation des tâches, formaliser clairement le contenu des tâches \\
\hline Epuisement & $\begin{array}{l}\text { Exemple: Réduction des tâches, Donner la possibilité de choisir les moments } \\
\text { de pause, prévoir et aménager des espaces de détentes. }\end{array}$ \\
\hline
\end{tabular}

\section{Variation de la criticité des RPS}

Les employés interrogés perçoivent que les risques psychosociaux les plus importants pour eux sont le stress au travail et l'épuisement professionnel (sur une liste de stress, violence, harcèlement, souffrance au travail, épuisement professionnel). Selon les employés : "les facteurs du stress peuvent en plus apparaître au niveau du bruit, les conditions de chaleur et d'humidité, les vibrations et les expositions nocifs, etc ...."Les employés comme leur organisation sont convaincu que le rôle de la démarche RSE est d'améliorer le bien être au travail, mais ils affirment 
qu'elle reste insuffisante pour réduire le RPS ..."Il faut agir en plus sur d'autres facteurs liés à la vie quotidienne" Sa contribution est située entre $20 \%$ et $40 \%$. Le tableau suivant fait la synthèse de la variation de la criticité des RPS suite à une démarche RSE:

Tableau 6: Criticité avant la RSE et après la RSE

\begin{tabular}{|l|l|l|l|l|l|l|l|l|}
\hline RPS & \multicolumn{9}{|l|}{ Avant la RSE } & $\begin{array}{l}\text { Après la RSE } \\
\text { ce }\end{array}$ & $\begin{array}{l}\text { Fréquen } \\
\text { té }\end{array}$ & $\begin{array}{l}\text { Détectabi } \\
\text { lité }\end{array}$ & $\begin{array}{l}\text { Critici } \\
\text { té }\end{array}$ & $\begin{array}{l}\text { Fréquen } \\
\text { ce }\end{array}$ & $\begin{array}{l}\text { Gravi } \\
\text { té }\end{array}$ & $\begin{array}{l}\text { Détectabi } \\
\text { lité }\end{array}$ & $\begin{array}{l}\text { Critici } \\
\text { té }\end{array}$ \\
\hline Stress & 4 & 2 & 3 & 24 & 3 & 2 & 2 & 12 \\
\hline Violence & 1 & 3 & 1 & 3 & 1 & 2 & 1 & 2 \\
\hline $\begin{array}{l}\text { Harcèlem } \\
\text { ent }\end{array}$ & 2 & 2 & 2 & 8 & 2 & 1 & 2 & 4 \\
\hline $\begin{array}{l}\text { Epuiseme } \\
\text { nt }\end{array}$ & 3 & 2 & 2 & 12 & 2 & 1 & 1 & 2 \\
\hline
\end{tabular}

Les écarts relevés au niveau de la criticité des RSP sont expliqués par la démarche RSE selon les employés et les responsables. En réalité, la démarche leur permet de rendre le travail convenable aux conditions des employés, améliorer l'ambiance entre les collègues, d'augmenter le niveau de confiance entre les employés, améliorer les relations avec les supérieurs, justifier la quantité du travail fournis, perfectionner la manière d'organisation de travail, améliorer la manière dont les décisions sont prises, personnaliser le niveau de compétence demandé par chaque employé, augmenter l'autonomie donnée au employés, donner la flexibilité des horaires, bien prévoir le travail fais dans l'urgence.

La RSE permet d'agir donc sur le stress (criticité 12) d'abord en facilitant sa détectabilité tout en identifiant les indicateurs clés de son déclenchement, et puis elle consiste à adapter les compétences des employés avec les tâches qu'on leur demande, ce qui permet et réduire le niveau de décalage existant entre la perception des exigences et des compétences.

Pour l'épuisement professionnel (criticité 2), il est traité par la RSE en proposant plus de flexibilité des horaires au travail, par l'augmentation de l'autonomie et par la restructuration et le remodelage des tâches. les politiques de recrutement et les politiques de la formation continue pratiquées par l'ANP jouent aussi un rôle important pour assister les employés à dépasser l'épuisement professionnel.

\section{Conclusion}

En guise de conclusion, il est important de retenir que les RPS s'inscrivent dans une logique systémique de facteurs en interaction ayant un caractère fortement probabiliste. Ainsi, en analysant l'apport social de la RSE pour mettre en lumière la relation émergente entre cette dernière et la gestion des RPS, nous avons constaté sa forte contribution par ses approches 
préventives et correctives à la maîtrise des facteurs des RPS et à l'amélioration du bien être au travail.

Le travail a commencé par la présentation des fondements théoriques de la démarche RSE et de son déploiement, puis il a explicité une analyse des RPS, pour en troisième étape croiser la méthodologie et l'action de la RSE sur les facteurs générateurs des RPS.

Côté terrain, et en se basant sur les l'exploitation des résultats de l'enquête (trois questionnaires administrés chez l'ANP) sur la perception des salariés de la démarche et sur sa contribution à la réduction de leur RPS, nous avons présenté l'état des lieux du déploiement et de la maturité de la RSE chez l'ANP à travers le test de matérialité, puis nous avons présenté une évaluation du volet social de la démarche à travers la méthode des scores, puis en fin nous avons analysé les niveaux de criticité des RPS avant et après le déploiement de la démarche de RSE depuis 2014.

En termes de perspectives, nous nous posons la question de recherche pour comprendre pourquoi les pratiques de RSE constituent-elles des facteurs de stress pour les profils supérieurs dans une organisation?

\section{References:}

ACQUIER A., GOND J.P.( 2005). "Aux sources de la Responsabilité Sociale de l'Entreprise: relecture et analyse d'un ouvrage séminal : Social Responsibilities of the Businessman d'Howard Bowen (1953) », XIVème Conférence de l'Association internationale de management stratégique (AIMS).

ARACT Martinique ELVIE 2009 "Une méthode de diagnostic et de prévention de risques psychosociaux au travail" Edition ARACT 2009.

ANACT : Etudes et documents " Prévention du stress et des risques psychosociaux au travail », éditions réseau ANACT, 106 p., 2008

ANACT, 2010 "Risques psychosociaux : repères pour la négociation d'un accord" mars 2010.

BEN YEDDER, \& ZADDEM, (2009) La Responsabilité Sociale de l'Entreprise (RSE), voie de conciliation ou terrain d'affrontements? Revue multidisciplinaire sur l'emploi, le syndicalisme et le travail (REMEST), 2009, vol. 4, No 1.

CAPRON M. (2003). « L’économie éthique privée: La responsabilité des entreprises à l'épreuve de l'humanisation de la mondialisation », Economie Ethique, No7. pp 1-72. C

CAPRON M., QUAIREL-LANOIZELEE F. (2007). La responsabilité sociale d'entreprise, Paris, La Découverte, 122 p.

Carroll, A. B., \& Shabana, K. M. (2010) The Business Case for Corporate Social Responsibility: A Review of Concepts, Research and Practice. International Journal of Management Reviews, 12(1), 85-105. 
Conne-Perréard et Glardon M. Massimo J. Usel, 2001, "Effets de conditions de travail défavorables sur la santé des travailleurs et leurs conséquences économiques" conférence romande et tessinoise des offices Cantonaux de protection des travailleurs 2001.

COMMISSION DES COMMUNAUTES EUROPEENNES. (2001). Livre vert, promouvoir un cadre européen pour la responsabilité sociale des entreprises, Bruxelles, $31 \mathrm{p}$.

CHOUANIERE D. "Stress au travail une réalité ... quelle prévention, quels acteurs et quels outils ?" 2007 ;

CEE, "Transformations du travail et dialogue social", FGMM-CFDT, mai 2012. CEE, 2006.

DARES 2008, "Les facteurs psychosociaux au travail : une évaluation par le questionnaire de KARASEK dans l'enquête Sumer $2003 \mathrm{~N}^{\circ} 22.1$.

DOH J.P., GUAY T.R. (2006). “Corporate Social Responsibility, Public Policy, and NGO Activism in Europe and the United States: An InstitutionalStakeholder Perspective”, Journal of Management Studies, Vol 43, $\mathrm{n}^{\circ} 1$, pp 47-73.

EUROGIP47/F "Risques psychologiques au travail: une problématique européenne", 2010.

EU-OSHA. 2003, Comment maîtriser les problèmes psychosociaux et réduire le stress d'origine professionnelle, 2003,

Elodie Montreuil 2014 " Un lean mal accompagné génère des RPS", La Revue des conditions de travail p: 47.

European Agency for Safety and Health at Work 2013 Gender issues in safety and health at work A review ISBN 92-9191-045-7

Fraisse A, Guerfel-Henda S. 2005, La Responsabilité Sociale de l'Entreprise (RSE) : instrument de management des ressources humaines?" 16e Conférence de l'AGRH 2005.

IGALENS J., GOND J.P. (2003). "La mesure de la performance sociale de l'entreprise : une analyse critique et empirique des données ARESE", Revue Française de Gestion des Ressources Humaines, Iss. 50, pp. 111-130.

IGALENS, J. et TAHRI, N (2012), « Perception de la RSE par les salariés : construction et validation d'une échelle de mesure ", Revue de Gestion des Ressources Humaines, Nº3 Janvier Fevrier -Mars 2012, p. 3-19.

IVANAJ V., MCINTYRE J. (2006). " Multinational enterprises and sustainable development: a review of strategy process research», Colloque international "Multinational Enterprise and Sustainable Development: Strategic Tool for Competitiveness" USA.

IRES, "Modes de gestion des restructurations", IRES, n 72, 2012.

International Organization for Standardization 2010, ISO 26000 project overview ISBN 978-92-67-10537-6 
INRS 2013 et 2014: Etudes et recherches Edition Institut National de recherche et sécurité.

LOCKETT A., MOON J., VISSER W, (2006) “Corporate social responsibility in management research: focus, nature, salience and sources of influence”, Journal of Management Studies, Vol 43. №1, pp 115-35.

Leroux E. et Van de Portal M. (2011), « La perception du rôle de la GRH par les salariés dans l'approche des risques psycho-sociaux en entreprise », Humanisme et entreprise, $\mathrm{n}^{\circ}$ 301,p.

MANZANO M. (2010), «Les « risques psychosociaux » et le psychologue du travail... en Service de santé au travail (SST) », Nouvelle revue de psychosociologie, $\mathrm{n}^{\circ} 10$, p. 179-193.

Mathieu Pierre, (2008), "La RSE : Fondements théoriques et problématiques gestionnaires". Entreprise Ethique, Association Francophone de Comptabilite, 2008, pp.13-21.

MITCHELL, R.B.R. AGLE and D.J.WOOD (1997), " Toward a theory of stakeholder identification and salience: Defining the principle of who and what rally counts », Academy of Management Review, vol.22, N 4, pp.853886

Ndjaboué Ruh, Michel Vézina et Chantal Brisson 2012, "Effects of psychosocial factors at work on mental health. A literature review of prospective studies on three emerging models" Revue Travail et Emploi 129 janvier - mars 2012.

RAMBAUD A, MARNEFEE T. (2009) «Avis d'experts » in Quelle place pour la mobilisation des salariés dans les rapports de développement durable des entreprises du CAC40, Novethic 2009

TAHRI. N. (2010), Les effets psychosociologiques des pratiques socialement responsables sur les comportements des salariés au travail, Revue internationale de psychosociologie.

Technolgica 2013 "Guide pratiques des risques psychologiques" Edition mars 2013.

SAUTRE G. (2003). « Les balises éthiques en milieu de travail : nouvelles contraintes ou nécessité », Revue internationale des relations de travail, Vol 1, $\mathrm{N}$ 2, pp 37-67.

Wassenhove.W.V 2014 "Modèle de Karasek" sous la direction de Philippe Zawieja et Franck Guarnieri. Dictionnaire des risques psychosociaux, Le Seuil, pp.170-174, 978-2-02-110922-

WOOD D. J. 1991. 'Corporate social performance revisited', Academy of management Review, Iss. 16, pp 691-718.

WINDSOR D. 2006. "Corporate social responsibility: three keys approaches”, Journal of Management Studies, Vol 43, N 1, pp 93-115. 